\title{
Quantitative evaluations of ankle spasticity and stiffness in neurological disorders using manual spasticity evaluator
}

\author{
Qiyu Peng, PhD; ${ }^{1}$ Hyung-Soon Park, PhD; ${ }^{1-3}$ Parag Shah, MD; ${ }^{1}$ Nicole Wilson, PhD; ${ }^{1}$ Yupeng Ren, MS; ${ }^{1}$ \\ Yi-Ning Wu, PhD; ${ }^{1}$ Jie Liu, BS; ${ }^{1,4}$ Deborah J. Gaebler-Spira, MD; ${ }^{1-2}$ Li-Qun Zhang, PhD ${ }^{1-2,4^{*}}$ \\ ${ }^{1}$ Rehabilitation Institute of Chicago, Chicago, IL; ${ }^{2}$ Department of Physical Medicine \& Rehabilitation, Northwestern \\ University, Chicago, IL; ${ }^{3}$ Clinical Research Center, Rehabilitation Medicine Department, National Institutes of Health, \\ Bethesda, MD; ${ }^{4}$ Department of Biomedical Engineering, Northwestern University, Chicago, IL
}

\begin{abstract}
Spasticity and contracture are major sources of disability in people with neurological impairments that have been evaluated using various instruments: the Modified Ashworth Scale, tendon reflex scale, pendulum test, mechanical perturbations, and passive joint range of motion (ROM). These measures generally are either convenient to use in clinics but not quantitative or they are quantitative but difficult to use conveniently in clinics. We have developed a manual spasticity evaluator (MSE) to evaluate spasticity/contracture quantitatively and conveniently, with ankle ROM and stiffness measured at a controlled low velocity and joint resistance and Tardieu catch angle measured at several higher velocities. We found that the Tardieu catch angle was linearly related to the velocity, indicating that increased resistance at higher velocities was felt at further stiffer positions and, thus, that the velocity dependence of spasticity may also be position-dependent. This finding indicates the need to control velocity in spasticity evaluation, which is achieved with the MSE. Quantitative measurements of spasticity, stiffness, and ROM can lead to more accurate characterizations of pathological conditions and outcome evaluations of interventions, potentially contributing to better healthcare services for patients with neurological disorders such as cerebral palsy, spinal cord injury, traumatic brain injury, and stroke.
\end{abstract}

Key words: catch angle, cerebral palsy, contracture, manual spasticity evaluator, neurological disorders, quantitative measurement, range of motion, spasticity, Tardieu scale, velocity.

\section{INTRODUCTION}

People with injuries such as stroke, cerebral palsy (CP), and traumatic brain injury (TBI) often experience residual physical impairments such as spastic hypertonia and muscle contracture [1-7]. Spasticity is a major source of disability in these patients. Hypertonus and reflex hyperexcitability disrupt the remaining functional use of muscles, impede motion, and may cause severe pain. Prolonged spasticity may be accompanied by structural changes in muscle fibers and connective tissue, which may reduce joint range of motion (ROM) and lead to clinical contracture $[4,8]$.

Accurate and reliable evaluation of spasticity presents a constant challenge to the medical rehabilitation community. The Modified Ashworth Scale (MAS) is the most popular clinical measure of spasticity [9]; however,

\footnotetext{
Abbreviations: $\mathrm{CP}=$ cerebral palsy, ICC $=$ intraclass correlation coefficient, MAS = Modified Ashworth Scale, MSE = manual spasticity evaluator, $\mathrm{ROM}=$ range of motion, $\mathrm{SCI}=$ spinal cord injury, TBI = traumatic brain injury.

* Address all correspondence to Li-Qun Zhang, PhD; Rehabilitation Institute of Chicago, 345 E Superior Street, Suite 1406, Chicago, IL 60611; 312-238-4767; fax: 312-238-2208. Email: 1-zhang@northwestern.edu

DOI:10.1682/JRRD.2010.04.0053
} 
poor inter- and intrarater reliability of the MAS has been reported [10-13]. The Tardieu scale is another clinical measure of spasticity that compares how spastic muscles "catch" at low, medium, and high velocities [14]. The Tardieu scale has better reliability than the MAS when raters receive identical and intensive training [15], which implies that standardized training would improve reliability among raters who receive training from different trainers.

In general, clinical scales are practical and convenient to use in clinics but less accurate than quantitative measurements. A handheld dynamometer or spring scale has been used to measure the force rotating the limb during spasticity testing in the clinic [16-17]. More sophisticated robotic devices have been used to obtain the torque-angle curve, from which joint ROM, (quasi-static) stiffness, and energy loss can be derived [18-20]. The use of computer-controlled devices, however, adds procedures to existing assessment protocols, making the protocols more sophisticated, which may prevent clinicians from using them in clinical practice.

This article presents a manual spasticity evaluator (MSE) that can be used conveniently by clinicians and that enables quantitative evaluation of spasticity and contracture at the ankle joint. The MSE was tested to measure ankle ROM at controlled low velocity, elastic stiffness, spasticity, and Tardieu catch angle at higher velocities.

\section{METHODS}

\section{System Configuration}

The MSE (Figure 1) consists of a custom-designed foot plate with a torque sensor (TRT-200; Transducer Techniques Inc; Temecula, California), a position sensor, a handle to move the foot plate, a leg support, and a laptop with data acquisition card (NI DAQ card 6036E; National Instruments Co; Austin, Texas).

A custom data-acquisition program was developed to implement two functions: (1) measure, record, and display both the torque signal and the position signal and (2) produce real-time audio-visual feedback when a preset velocity and/or torque limit was reached. With the help of the real-time record of torque and position signals and audio-visual feedback, the examiner can characterize the pathological conditions more accurately by measuring the passive ROM at consistent peak torques, resistance torque at fixed positions, elastic stiffness, and catch angles and, in addition, have the capability to display and

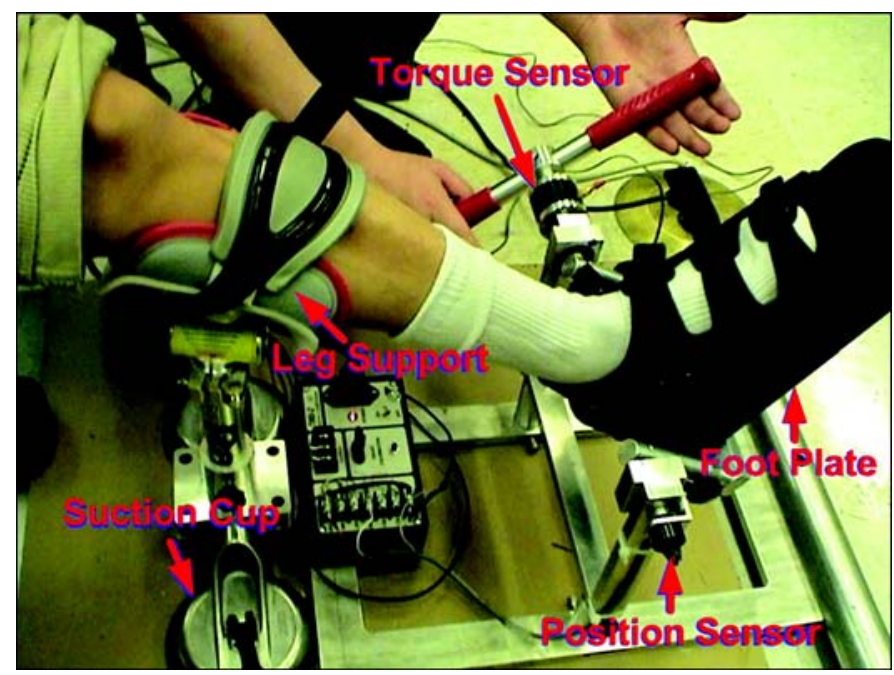

Figure 1.

Manual spasticity evaluator used to measure spasticity at human ankle. With subject's leg fixed to leg support, operator moves ankle at controlled velocity and terminal torque with real-time feedback. Ankle movement and joint torque measured by position sensor and torque sensor, respectively.

analyze the recorded data in different formats (e.g., angular position and torque signals as functions of time and torque-angle hysteresis loops).

\section{Human Subjects and Experimental Procedures}

Twelve children with CP aged 4 to $19 \mathrm{yr}(12.0 \pm 5.0 \mathrm{yr})$ who had ankle spasticity and five nondisabled children aged 12 to $14 \mathrm{yr}(12.0 \pm 1.4 \mathrm{yr})$ were recruited (all data presented as mean \pm standard deviation unless otherwise noted). In addition, five nondisabled adults aged 21 to $31 \mathrm{yr}$ (24.4 $\pm 3.7 \mathrm{yr}$ ) were recruited to determine the intra- and interrater reliability of the MSE system.

The following procedure was performed to test passive ROM and spasticity. First, the subject's foot was placed on the foot plate and the subject's ankle joint was aligned with the rotation axis of the device by sliding the foot plate in anterior/posterior and proximal/distal directions. Then, the foot was fixed to the plate with Velcro straps. The operator then manually moved the ankle joint from one extreme position to the other at a constant velocity. In this procedure, the laptop sampled and displayed the joint angular position and resistance torque (sampling rate: 1,000 $\mathrm{Hz}$ ). When the desired terminal torque or target velocity was reached, the software alerted the operator with audio-visual displays (Figure 2). The operator repeated the passive motion tests at various 


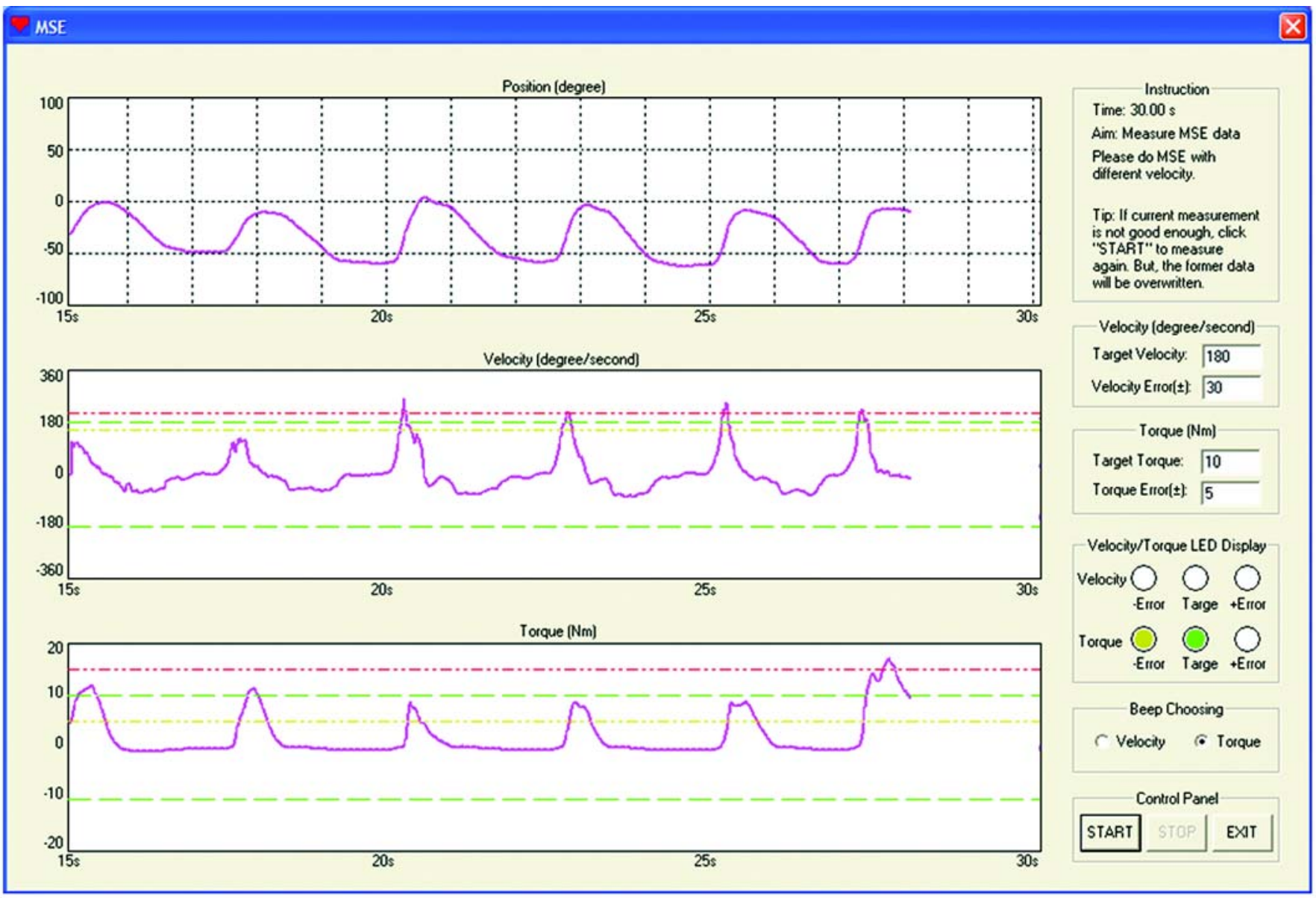

Figure 2.

Screen display of data acquisition and test-control program. From top to bottom, plots show real-time ankle dorsiflexion angle, velocity, and joint torque, respectively. Their target values and ranges can be seen as horizontal lines, and beeping sound is generated once target is within range. Simulated light-emitting diode buttons also indicate when target torque or velocity has been reached within certain range.

velocities in both the dorsal and plantar directions. The position and torque data were recorded and then analyzed offline to calculate variables, including the Tardieu catch angle, for the quantitative assessment of the neuromuscular and biomechanical properties of the joints.

Note that the target terminal torque and the target velocity shown by the broken lines in Figure 2 were used only to guide and inform the operator. To improve the quality of data obtained, the operator practiced before collecting actual data. Usually, the data collected were reliable and repeatable (as shown in Figure 3). However, large artifacts could also be introduced if the subject moved his or her ankles during the testing (shown in the last plot in Figure 2). We therefore developed custom MATLAB (The MathWorks, Inc; Natick, Massachusetts) programs to help the operator visually screen and inspect the data immediately after a trial. With the help of those programs, the operator could include or exclude a specific trial according to the inspection.

\section{Tardieu Catch Angle}

Tardieu scales were measured quantitatively and conveniently with the MSE. As shown in a typical torqueangle curve from a child with $\mathrm{CP}$, the position at which the torque increased most sharply (highest torque change rate, indicated by red circle in Figure 4) was determined as the catch angle, i.e., the position at which the operator felt the sudden increase in resistance - the "catch."

Four typical curves (torque, time derivative of the torque, instant velocity, and position) were used to determine the Tardieu catch angle (Figure 5). 


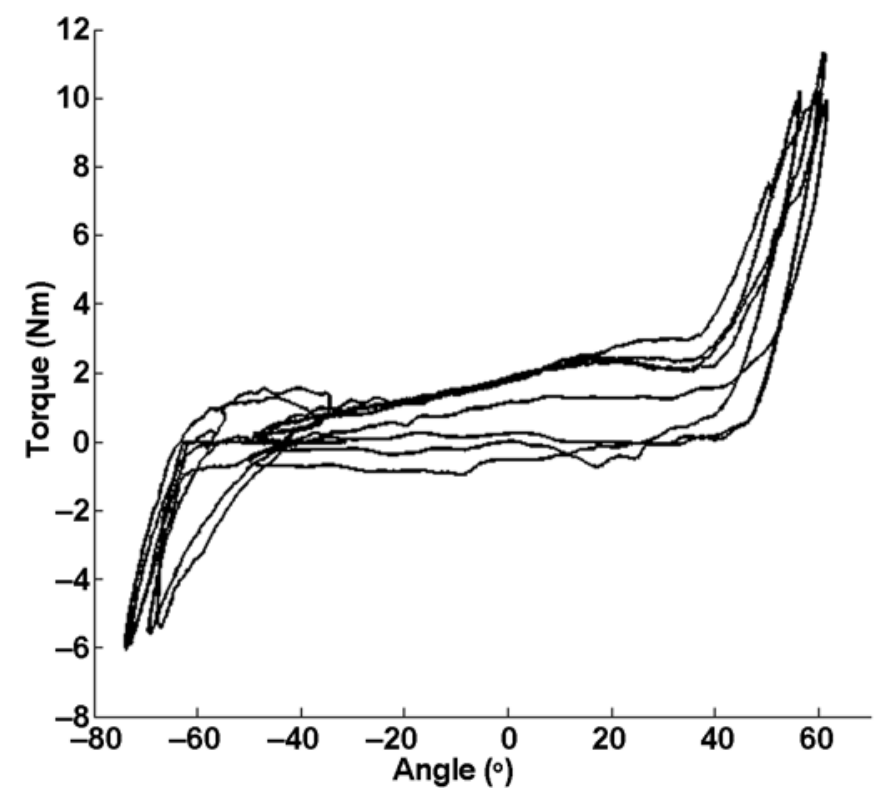

Figure 3.

Typical torque-angle curve from nondisabled child. Four trials presented in this curve.

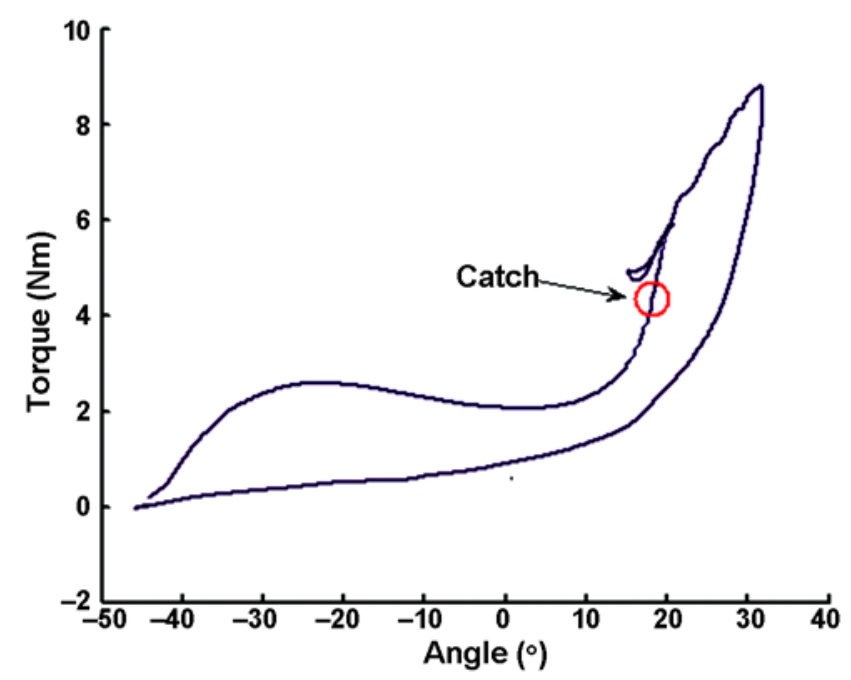

Figure 4.

Typical torque-angle curve with catch from child with cerebral palsy.

The waveforms in Figure 5 imply that the Tardieu catch angle can be identified by three characteristics: (1) the time derivative of the torque ( $\Delta$ torque/ $\Delta t$ ) increases to a peak value (position 1 in Figure 5(a), time point 1 in Figure 5(b)) and then decreases to a minimum value, (2) the position-torque curve moves backward shortly

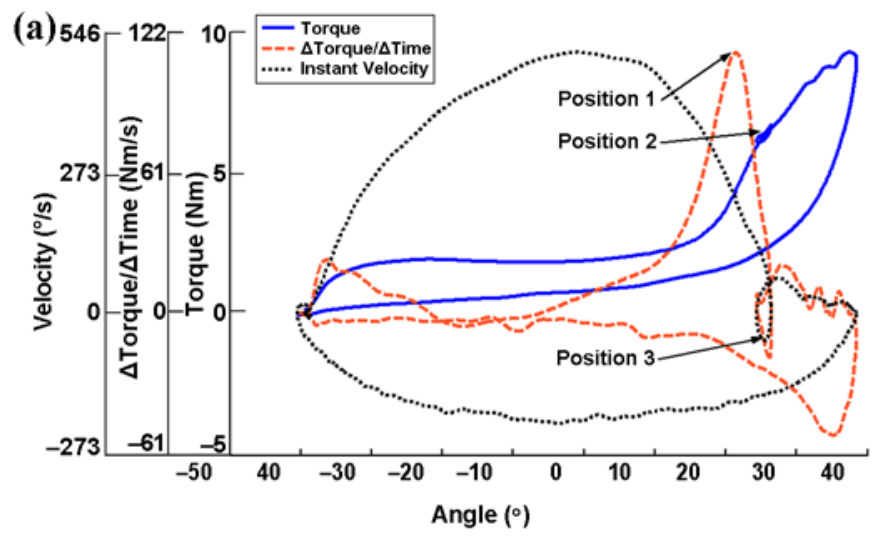

(b)

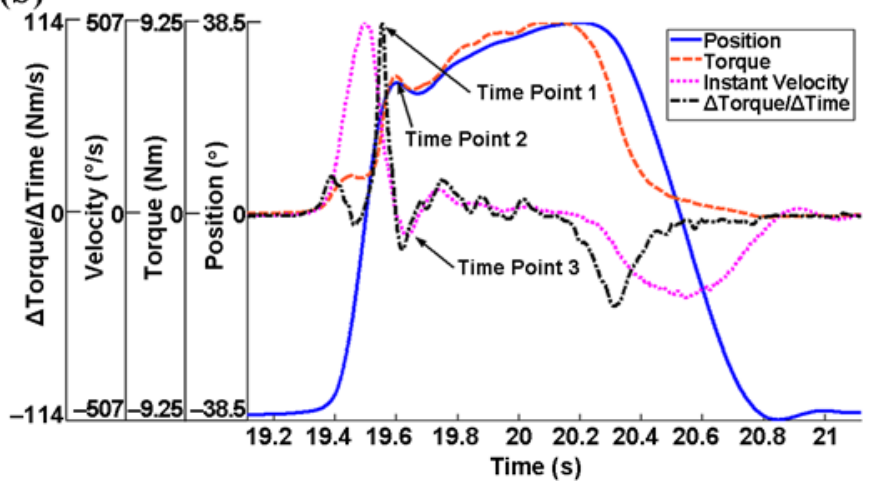

Figure 5.

Typical torque and position signals recorded using the manual spasticity evaluator. (a) $x$-axis represents ankle dorsiflexion angle. Solid, dashed, and dotted waveforms are torque, velocity, and $\Delta$ torque/ $\Delta$ time shown as functions of joint angle, respectively. (b) Solid, dashed, dotted, and dot-dashed waveforms are torque, velocity, position, and $\Delta$ torque/ $\Delta$ time shown as functions of time, respectively.

then moves forward (position 2 in Figure 5(a), time point 2 in Figure 5(b)), and (3) velocity decreases to a minimum and then increases (position 3 in Figure 5(a), time point 3 in Figure 5(b)).

Three characteristics are associated with the catch phenomenon: (1) the examiner feels the sharp increase of the resistance torque (peak of the time derivative of the torque: position 1 in Figure 5(a), time point 1 in Figure 5(b)) when the catch phenomenon occurs. (2) The operator then responds to the sharp increase in torque by decreasing the velocity. If the velocity is decreased to negative, the position-torque curve moves backward (position 2 in Figure 5(a), time point 2 in Figure 5(b)). (3) As the operator decreases the velocity, the time derivative of the torque decreases. Then, the operator increases the velocity in the forward direction up to the extreme position. This causes the second increase in velocity after 
the minimum is reached (position 3 in Figure 5(a), time point 3 in Figure 5(b)).

According to these observations, both time point 2 in the position-torque curve and time point 3 (Figure $5 \mathbf{b}$ ) in the velocity-angle curve are caused by the sharp increase of the resistance torque (time derivative of the torque); the angle at which the time derivative of the torque reaches the maximum is considered the catch angle.

\section{Statistical Analysis}

To determine the intra- and interrater reliability of the MSE system, we recruited five nondisabled adults. Two raters performed MSE testing on them twice. The intraclass correlation coefficient (ICC) was chosen as the test statistic with which we evaluated test-retest reliability. The two-way mixed model ICC was used. ICCs of 0.75 or more indicated excellent reproducibility. The two rater's scores were compared with Pearson product-moment correlations.

\section{RESULTS}

\section{Range of Motion and Stiffness}

Ankle ROM and elastic stiffness were measured at a controlled low velocity (about $30^{\circ} / \mathrm{s}$ ). For children with $\mathrm{CP}$ and limited ankle movement, the ROM (ROM1 in Figure 6) could be markedly smaller than that of nondisabled children (ROM2 in Figure 6). Across all the subjects, the total mean passive ROM for the CP and control groups was $93.4^{\circ} \pm 24.6^{\circ}$ and $94.7^{\circ} \pm 24.0^{\circ}$, respectively. However, the difference was not statistically significant ( $p=0.9$ ), which may be related to the small sample size and/or the wide age ranges of the participants in this study.

The intra- and interrater reliability test showed that this measure has high reproducibility (ICC $=0.86$, Pearson $r=0.95, p<0.001$ ).

All participants in this study had a ROM that included the position of $4^{\circ}$ of dorsiflexion. We therefore measured stiffness at this angle to compare the two groups. As shown in the examples in Figure 6, joint stiffness at $4^{\circ}$ of dorsiflexion was greater for the children with CP (CP.Stiff in Figure 6) than the stiffness of the nondisabled children (N.Stiff in Figure 6). Overall, the stiffness at the given ankle position in children with CP was higher than that of nondisabled children, with mean ankle stiffness for the $\mathrm{CP}$ and control groups of $0.25 \pm$ 0.27 and $0.10 \pm 0.11 \mathrm{Nm} / \mathrm{rad}$, respectively. However, the

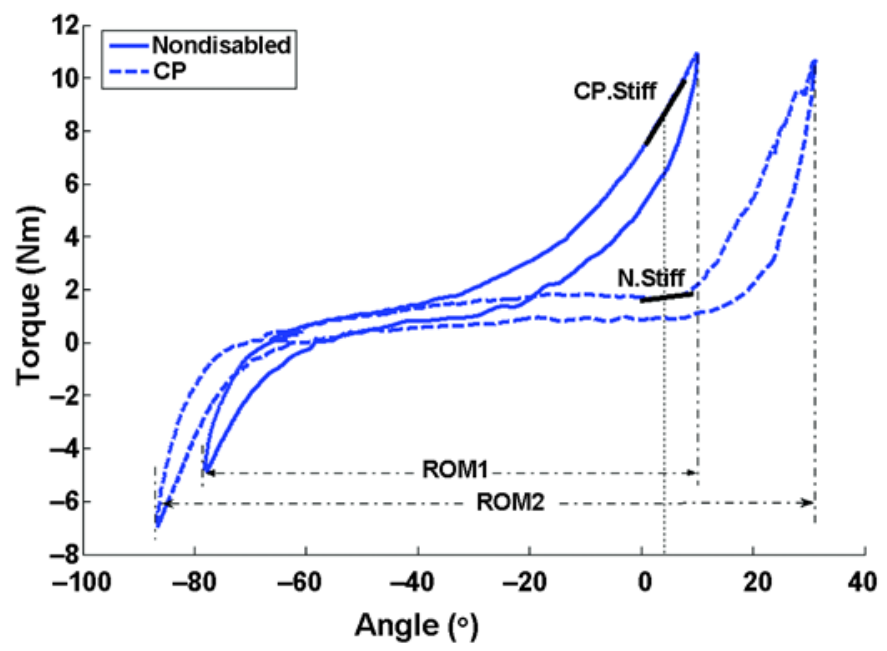

Figure 6.

Typical torque-angle curves (hysteresis loops) from child with CP (solid curve; CP.Stiff) and nondisabled (dashed curve; N.Stiff) subjects: $x$ - and $y$-axes are dorsiflexion angle and passive resistance torque, respectively. $\mathrm{CP}=$ cerebral palsy, $\mathrm{ROM}=$ range of motion, ROM1 = ROM of children with CP and limited ankle movement, ROM2 = ROM for nondisabled children.

difference was not statistically significant $(p=0.3)$, which may be related to the small sample size and/or the wide age ranges of the participants in this study.

The intra- and interrater reliability test showed that this measure has high reproducibility (ICC $=0.82$, Pearson $r=0.81, p=0.002$ ).

\section{Tardieu Catch Angle and Velocity}

Linear correlation between Tardieu catch angle and velocity of children with CP was found (Figure 7).

\section{DISCUSSION AND CONCLUSIONS}

Hypertonia is a major source of disability in patients with neurological disorders including $\mathrm{CP}$, stroke, spinal cord injury (SCI), and TBI and is characterized by spasticity and/or contracture in the involved joints. The associated changes in the mechanical properties of muscles and tendons may reduce joint ROM and limb deformity. Accurate and reliable evaluation of spasticity is essential for diagnosis, treatment, and patient management (determining therapy and evaluating treatment outcomes). In this study, we developed a MSE and demonstrated its efficacy. It is important to point out that the MSE is not 


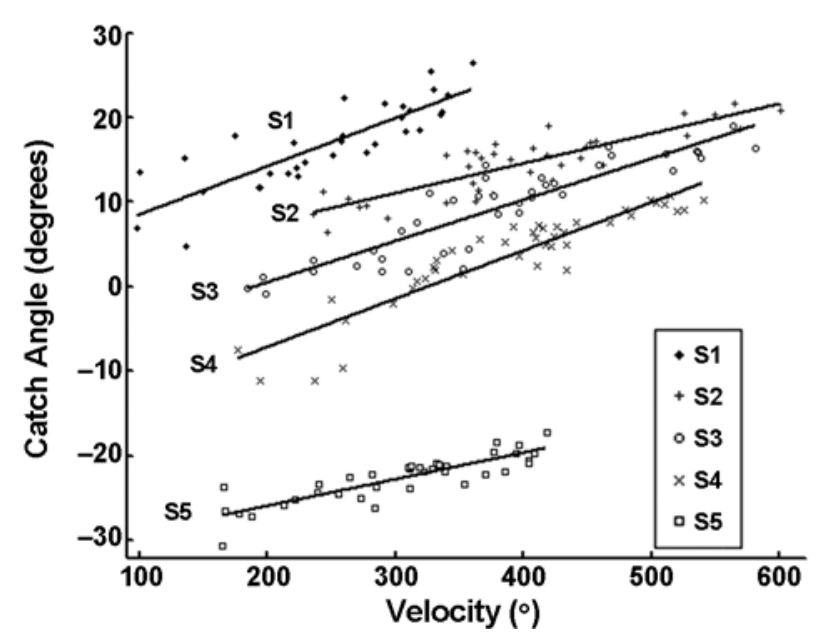

Figure 7.

Relationship between dorsiflexion velocity and catch angle of five children with cerebral palsy: $x$-axis is velocity, and $y$-axis is Tardieu catch angle. Solid lines are linear fit of raw data. S1 to S5 each correspond to data from subjects $1-5$.

designed exclusively for children. It can also be applied to characterize spasticity/contracture in adults with neurological disorders (e.g., SCI, TBI, and stroke).

Children with CP have alterations in the biomechanical and reflex properties of the ankle. These changes have been assessed by such measures as the MAS, tendon reflex scale, pendulum test, mechanical perturbations, and passive joint ROM. Manual assessments through physical examination by experienced clinicians are intrinsically subjective and qualitative and may have poor intra- and interrater reliability. Sophisticated robotic devices can accurately measure the torque-angle curve, joint ROM, stiffness, and energy loss. However, they are usually not convenient to use in clinical settings. Haptic robotic devices have been used to re-create the sensations clinicians would have felt during an in-person examination [21]; however, re-created haptic sensations are not identical to the actual feeling, which is important in clinical examinations.

In this study, we designed an MSE that can be used to evaluate spasticity and stiffness quantitatively and conveniently. The MSE provides clinicians with both transparent haptic feedback and quantitative measurements of the biomechanical and reflex properties of the ankle under controlled resistance torque and/or velocity.

Using the MSE, we tested 12 children with CP and 5 nondisabled children. We measured the ankle ROM and stiffness at controlled low velocity, velocity-dependent resistance, and Tardieu catch angle at several different velocities in a clinical setting. The results show that the Tardieu angle, ROM, and stiffness in the spastic ankle can be determined quantitatively and conveniently. Compared with the control group, the CP group had higher joint stiffness, while no difference was noted in passive ROM between the two groups. We also found that the catch angle was linearly dependent on the movement velocity and thus should be determined at a well controlled velocity for consistent results. The linear relation between movement velocity and catch angle was also found in a related study on the upper limb [22].

We also tested five nondisabled adults to determine the intra- and interrater reliability of the MSE system. The results show that the MSE system has excellent reproducibility in passive ROM (ICC $=0.86$, Pearson $r=$ $0.95, p<0.001$ ) and stiffness measurements (ICC $=0.82$, Pearson $r=0.81, p=0.002$ ).

The catch angle is conventionally defined as the position at which the tested muscle reacts to stretching $[10,14]$. However, the measurement is dependent on the examiners' experience. Therefore, conventional determination of the catch angle is intrinsically subjective. We therefore chose the maximum rate-of-increase point of the torque as the catch angle. This seems consistent with how clinicians determine the catch-when they feel a sudden increase in resistance. Our experimental results show that the definition is objective and consistent with the examiner's haptic feeling.

Velocity dependence of joint resistance during passive movement is a key feature of spasticity. Nonetheless, the so-called velocity dependence of spasticity can also be confused with position dependence. The catch angle occurring at a further position at higher velocities in our experiment demonstrated that spasticity is also position dependent, since the further position is associated with higher resistance. Namely, with increasing velocity, the joint was moved more rapidly to positions of higher resistance torque, as felt by the examiner [22]. Furthermore, the position dependence is related to the time delay associated with neurological reflexes. Generally, the reflex loop from muscle stretch to torque generation has a time delay of $\sim 40-60 \mathrm{~ms}$, depending on the joint and conditions $[2,23]$. This joint movement during the reflex time delay seems to correlate well with the velocity increases in Figure 7. When comparing the velocities of $200^{\circ} / \mathrm{s}$ and $400^{\circ} \mathrm{s}$, one finds that an extra $10^{\circ}$ is traveled at the 
higher velocity ( $400 \%$ s) during the 50 ms reflex delay. In someone with a stroke, for example, the extra $10^{\circ}$ rotation at the higher velocity is usually associated with increased resistance.

The increased resistance torque could be from spastic responses and/or passive joint stiffness [2,24-25]. As a result, patients with only increased passive stiffness may be misdiagnosed with spasticity, which may then affect their clinical treatment. Therefore, evaluating spastic joints at various velocities may help us differentiate passive joint stiffness and spasticity [24]. Evaluations of joint stiffness and the Tardieu scale using the MSE can help us characterize spasticity and contracture quantitatively at controlled velocities in a clinical setting.

The MSE depends on the operator to provide constant velocity or constant torque perturbations. It is impossible for a human operator to achieve a constant velocity or torque as accurately as robotic devices do. When a clinician manually evaluates spasticity and moves the joint at low, medium, or high velocities, the velocity controlled is practically the peak movement velocity, and in manual tests, it cannot be quantitatively controlled. The MSE provides real-time audio-visual feedback and more accurate velocity control. Furthermore, the MSE can provide haptic feedback, which is important for clinicians. In constant-velocity experiments (Figure 2), the operator first held the MSE handle to keep the ankle at the neutral position for $\sim 1 \mathrm{~s}$. The velocity was zero during this period. Next, the operator stretched the ankle at a certain velocity. The MSE device provides audio-visual feedback to help the operator accurately achieve a peak velocity at the desired level. Operators can easily keep the peak velocity at the target range with the audio-visual feedback after several trials.

Limitations of the current study include the small sample size. The fact that no significant difference was found in the ROM between children with CP and nondisabled children did not necessarily mean there was no difference. It was likely that the small populations and the wide age range (6-19) for the two groups contributed to the lack of statistical significance. Another limitation was that clonus or multiple catch events (i.e., cogwheel rigidity [26]) were not investigated. These issues can be addressed in future studies.

In conclusion, measurement of ankle spasticity can be done more accurately and conveniently using a device like the MSE. More accurate measurements of spasticity and joint biomechanical properties can lead to more focused and impairment-specific interventions, such as botulinum toxin, physical therapy, and surgeries, potentially resulting in better healthcare services for patients with neurological disorders.

\section{ACKNOWLEDGMENTS}

\section{Author Contributions:}

Acquisition of data: Q. Peng, P. Shah, N. Wilson, J. Liu, L. Q. Zhang. Subject recruitment: D. J. Gaebler-Spira.

Analysis and interpretation of data: Q. Peng, P. Shah, N. Wilson, J. Liu, H. S. Park, Y. Ren, Y. N. Wu, L. Q. Zhang, D. J. Gaebler-Spira. Drafting of manuscript: Q. Peng, P. Shah, N. Wilson, J. Liu, H. S. Park, Y. Ren, Y. N. Wu, L. Q. Zhang, D. J. Gaebler-Spira. Critical revision of manuscript for important intellectual content: Q. Peng, P. Shah, N. Wilson, J. Liu, H. S. Park, Y. Ren, Y. N. Wu, L. Q. Zhang, D. J. Gaebler-Spira.

Statistical analysis: Q. Peng, P. Shah, N. Wilson, J. Liu, H. S. Park, Y. Ren, Y. N. Wu, L. Q. Zhang.

Obtained funding: L. Q. Zhang.

Study supervision: L. Q. Zhang.

Financial Disclosures: The authors have declared that no competing interests exist.

Funding/Support: This material was based on work supported with resources from the National Institutes of Health (grant R01HD044295), National Institute on Disability and Rehabilitation Research (grant H133S080074), and National Science Foundation (grants CBET-0854498 and IIP-0750515).

Institutional Review: This study was approved by the institutional review board of Northwestern University. All subjects gave informed consent, and for children under 18 years old, parental consent was obtained.

Participant Follow-Up: The authors do not plan to notify the study subjects of the publication of this article because of a lack of contact information.

\section{REFERENCES}

1. Allison SC, Abraham LD, Petersen CL. Reliability of the Modified Ashworth Scale in the assessment of plantarflexor muscle spasticity in patients with traumatic brain injury. Int J Rehabil Res. 1996;19(1):67-78. [PMID: 8730545] DOI:10.1097/00004356-199603000-00007

2. Chung SG, Van Rey EM, Bai Z, Rogers MW, Roth EJ, Zhang LQ. Aging-related neuromuscular changes characterized by tendon reflex system properties. Arch Phys Med Rehabil. 2005;86(2):318-27. [PMID: 15706561] DOI:10.1016/j.apmr.2004.04.048

3. Lance JW. Disordered muscle tone and movement. Clin Exp Neurol. 1981;18:27-35. [PMID: 6926389]

4. Rymer WZ, Katz RT. Mechanisms of spastic hypertonia. Phys Med Rehabil State Art Rev. 1994;8(3):441-54. 
5. Sherman KB, Goldberg M, Bell KR. Traumatic brain injury and pain. Phys Med Rehabil Clin N Am. 2006;17(2): 473-90,viii. [PMID: 16616278]

DOI:10.1016/j.pmr.2005.11.007

6. Young RR. Spasticity: A review. Neurology. 1994;44(11 Suppl 9):S12-20. [PMID: 7970006]

7. Zafonte R, Elovic EP, Lombard L. Acute care management of post-TBI spasticity. J Head Trauma Rehabil. 2004;19(2): 89-100. [PMID: 15247820] DOI:10.1097/00001199-200403000-00002

8. Bell KR, Vandenborne K. Contracture and limb deformities. In: Lazar RB, editor. Principles of neurologic rehabilitation. New York (NY): McGraw-Hill; 1998. p. 309-28.

9. Bohannon RW, Smith MB. Interrater reliability of modified Ashworth scale of muscle spasticity. Phys Ther. 1987; 67(2):206-7. [PMID: 3809245]

10. Mehrholz J, Wagner K, Meissner D, Grundmann K, Zange C, Koch R, Pohl M. Reliability of the Modified Tardieu Scale and the Modified Ashworth Scale in adult patients with severe brain injury: A comparison study. Clin Rehabil. 2005;19(7):751-59. [PMID: 16250194] DOI:10.1191/0269215505cr889oa

11. Sunnerhagen KS. Stop using the Ashworth scale for the assessment of spasticity. J Neurol Neurosurg Psychiatry. 2010;81(1):2. [PMID: 20019216]

DOI:10.1136/jnnp.2009.189068

12. Tederko P, Krasuski M, Czech J, Dargiel A, GarwackaJodzis I, Wojciechowska A. Reliability of clinical spasticity measurements in patients with cervical spinal cord injury. Ortop Traumatol Rehabil. 2007;9(5):467-83. [PMID: 18026067]

13. Yam WK, Leung MS. Interrater reliability of Modified Ashworth Scale and Modified Tardieu Scale in children with spastic cerebral palsy. J Child Neurol. 2006;21(12): 1031-35. [PMID: 17156693] DOI:10.1177/7010.2006.00222

14. Boyd RN, Graham HK. Objective measurement of clinical findings in the use of botulinum toxin type A for the management of children with cerebral palsy. Eur J Neurol. 1999;6(S4):s23-s35.

DOI:10.1111/j.1468-1331.1999.tb00031.x

15. Gracies JM, Burke K, Clegg NJ, Browne R, Rushing C, Fehlings D, Matthews D, Tilton A, Delgado MR. Reliability of the Tardieu Scale for assessing spasticity in children with cerebral palsy. Arch Phys Med Rehabil. 2010;91(3): 421-28. [PMID: 20298834]

DOI:10.1016/j.apmr.2009.11.017

16. Moseley A, Adams R. Measurement of passive ankle dorsiflexion: Procedure and reliability. Aust J Physiother. 1991; 37(3):175-81.

17. Tardieu C, Lespargot A, Tabary C, Bret MD. Toe-walking in children with cerebral palsy: Contributions of contracture and excessive contraction of triceps surae muscle. Phys Ther. 1989;69(8):656-62. [PMID: 2748720$]$

18. Hesse S, Schmidt H, Werner C, Bardeleben A. Upper and lower extremity robotic devices for rehabilitation and for studying motor control. Curr Opin Neurol. 2003;16(6): 705-10. [PMID: 14624080] DOI:10.1097/00019052-200312000-00010

19. Nef T, Quinter G, Muller R, Riener R. Effects of arm training with the robotic device ARMin I in chronic stroke: Three single cases. Neurodegener Dis. 2009;6(5-6):240-51. [PMID: 19940461] DOI:10.1159/000262444

20. Zhang LQ, Chung SG, Bai Z, Xu D, Van Rey EM, Rogers MW, Johnson ME, Roth EJ. Intelligent stretching of ankle joints with contracture/spasticity. IEEE Trans Neural Syst Rehabil Eng. 2002;10(3):149-57. [PMID: 12503779] DOI:10.1109/TNSRE.2002.802857

21. Park HS, Peng Q, Zhang LQ. A portable telerehabilitation system for remote evaluations of impaired elbows in neurological disorders. IEEE Trans Neural Syst Rehabil Eng. 2008;16(3):245-54. [PMID: 18586603]

DOI:10.1109/TNSRE.2008.920067

22. Wu YN, Ren Y, Goldsmith A, Gaebler D, Liu SQ, Zhang LQ. Characterization of spasticity in cerebral palsy: Dependence of catch angle on velocity. Dev Med Child Neurol. 2010;52(6):563-69. [PMID: 20132137] DOI:10.1111/j.1469-8749.2009.03602.x

23. Liu J, Ren Y, Xu D, Chung SG, Rymer WZ, Zhang LQ. Evaluation of neuromuscular dynamics of hyperactive reflexes at the elbow post stroke. Proceedings of the American Society of Biomechanics Annual Meeting; 2010 Aug 18-21; Providence, Rhode Island.

24. Damiano DL, Quinlivan JM, Owen BF, Payne P, Nelson KC, Abel MF. What does the Ashworth scale really measure and are instrumented measures more valid and precise? Dev Med Child Neurol. 2002;44(2):112-18.

[PMID: 11848107] DOI:10.1017/S0012162201001761

25. Singer BJ, Dunne JW, Singer KP, Allison GT. Velocity dependent passive plantarflexor resistive torque in patients with acquired brain injury. Clin Biomech (Bristol, Avon). 2003;18(2):157-65. [PMID: 12550815] DOI:10.1016/S0268-0033(02)00173-0

26. Sanger TD, Delgado MR, Gaebler-Spira D, Hallett M, Mink JW; Task Force on Childhood Motor Disorders. Classification and definition of disorders causing hypertonia in childhood. Pediatrics. 2003;111(1):e89-97.

[PMID: 12509602]

DOI:10.1542/peds.111.1.e89

Submitted for publication April 2, 2010. Accepted in revised form November 15, 2010. 
This article and any supplementary material should be cited as follows:

Peng Q, Park HS, Shah P, Wilson N, Ren Y, Wu YN, Liu J, Gaebler-Spira DJ, Zang LQ. Quantitative evaluations of ankle spasticity and stiffness in neurological disorders using manual spasticity evaluator. J Rehabil Res Dev. 2011;
48(4):473-82.

DOI:10.1682/JRRD.2010.04.0053

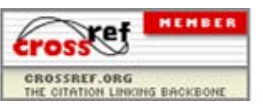


